\title{
Belphégor
}

\section{Jean-Pierre Galvan (éd.), Correspondance générale d'Eugène Sue. Volume II (1841-1845)}

\section{Vittorio Frigerio}

\section{(2) OpenEdition}

1 Journals

\section{Édition électronique}

URL : http://journals.openedition.org/belphegor/540

DOI : 10.4000/belphegor.540

ISSN : 1499-7185

Éditeur

LPCM

\section{Référence électronique}

Vittorio Frigerio, « Jean-Pierre Galvan (éd.), Correspondance générale d'Eugène Sue. Volume II

(1841-1845) », Belphégor [En ligne], 13-1 | 2015, mis en ligne le 09 mai 2015, consulté le 22 septembre 2020. URL : http://journals.openedition.org/belphegor/540 ; DOI : https://doi.org/10.4000/belphegor. 540

Ce document a été généré automatiquement le 22 septembre 2020.

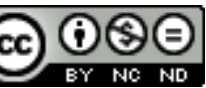

Belphégor est mis à disposition selon les termes de la Licence Creative Commons Attribution - Pas d'Utilisation Commerciale - Pas de Modification 4.0 International. 


\title{
Jean-Pierre Galvan (éd.), Correspondance générale d'Eugène Sue. Volume II (1841-1845)
}

\author{
Vittorio Frigerio
}

\section{RÉFÉRENCE}

Jean-Pierre Galvan (éd.), Correspondance générale d'Eugène Sue. Volume II (1841-1845).

Paris : Honoré Champion, 2013. 952 p. ISBN : 978-2-7453-2471. 
Que l'auteur des feuilletons les plus interminables $\mathrm{du}$ dix-neuvième siècle français ait été également un épistolier intarissable ne devrait guère surprendre. On ne peut toutefois retenir un mouvement de surprise, mâtiné d'admiration, devant l'épaisseur de ce second tome de la correspondance générale d'Eugène Sue, qui recueille l'ensemble des lettres qu'il a écrites pendant les années de sa popularité grandissante, et par conséquent pendant des périodes de travail créatif intense et exigeant.

Le dialogue, comme le fait remarquer justement Jean-Pierre Galvan dans son introduction, est toutefois justement l'essence de la composition romanesque telle que sue la conçoit. Il est au fondement d'un roman-feuilleton qui s'élabore et se construit en fonction des rapports que l'auteur entretient avec ses lecteurs et des lettres qu'il reçoit d'eux, et dont l'étendue dépend également de l'intérêt que le lectorat porte à l'ouvrage qui se forme ainsi au jour le jour devant ses yeux. Mais le feuilleton de Sue, Les Mystères de Paris notamment, qui se publie justement pendant la période que couvre ce volume de la correspondance de son auteur, s'écrit-il vraiment, comme le veut la doxa, au jour le jour et dans la hâte? Une des utilités, et pas la moindre, d'une immersion dans la vie quotidienne d'un écrivain telle que l'offre ce volume, est justement de permettre de nuancer, ou alors de contredire, un certain nombre de lieux communs que l'histoire littéraire finit par considérer vérités d'Évangile simplement du fait de leur répétition constante. Jean-Pierre Galvan peut ainsi, à travers l'analyse des lettres et des manuscrits, établir qu'en réalité le délai entre le moment de l'écriture et celui de la publication, pour la plus grande partie de l'œuvre, est d'à peu près un mois, et non pas de quelques petites heures comme le veut la légende. Cela lui permet notamment d'affirmer qu'«Eugène Sue ne bâcle jamais, il retravaille abondamment son texte et préfère suspendre la publication plutôt que de laisser insérer un feuilleton dont il n'a pas préalablement revu les épreuves » (p. 9). Cet auteur méticuleux, qui a conscience d'être en train de construire une œuvre littéraire, et qui deviendra avec ses Mystères et les romans qui suivront un des intellectuels progressistes les plus en vue de l'hexagone, a aussi souvent été accusé de s'être transformé en apôtre de la république démocratique et sociale sans conviction réelle, à la suite du succès populaire de son roman. Galvan rappelle comment l'éducation sociale de Sue, "placée sous l'influence de saint-simoniens, de fouriéristes et d'ouvriers éclairés » (p.11) n'aurait cependant pu avoir lieu sans une évolution morale préalable, dont les traces se retrouvent dans son œuvre bien avant le début de la publication des Mystères de Paris, qui fait de l'auteur dandy par excellence un philanthrope sensible aux malheurs des classes laborieuses. La correspondance permet également de mieux éclairer les rapports, parfois complexes et difficiles, du romancier et de ses éditeurs. 
3 Le volume est divisé par année, chaque année étant précédée d'une chronologie précise qui en résume les épisodes les plus marquants dans la vie et le travail de l'auteur. Les lettres sont abondamment et minutieusement annotées, ce qui permet de relier les événements narrés ou les opinions qui y sont exprimées avec tel ou tel passage d'un roman de Sue ou avec d'autres moments de sa carrière, ou de mieux comprendre des références à des personnages historiques, des ouvrages connus des auteurs, des articles de la presse, des missives, et bien d'autres choses encore. La précision, la profusion et l'utilité de ces notes ne sauraient être surestimées ni suffisamment louées. Un long «Dictionnaire des correspondants » fournit, en fin de volume, tous les renseignements nécessaires sur les personnes avec lesquelles l'auteur entretenait des rapports suivis.

4 Au-delà des nombreuses lettres qu'il échange avec ses éditeurs ou avec des personnalités et des membres de l'aristocratie et de la haute société européenne, qui sont toutefois loin d'être dénuées d'intérêt, les passionnés de littérature et les historiens du social trouveront leur bonheur à la lecture de la correspondance qui touche de plus près aux questions soulevées par la réception des Mystères de Paris par le public. L'ampleur du réseau de contacts de l'auteur - qui se retrouve à devoir entretenir des rapports suivis avec des légions de volontaires qui lui signalent des cas méritants sur lesquels se pencher ou qui débattent avec lui de la législation sociale des pays voisins et suggèrent des plans ou échafaudent des projets pour aider les classes défavorisées - est proprement ahurissante. Les mille projets pour obvier aux "déplorables lacunes» de la législation française et faire que la fiction ait un impact concret sur la réalité, les actes de charité commis par l'auteur sur le signalement de ses lecteurs (et surtout de ses lectrices, notamment Fanny Dénoix, inspectrice des écoles, et Ernestine Dumont, parmi les plus passionnées), parviennent aisément à convaincre que l'on ait pu à un moment donné estimer que Sue était «en France le seul justicier littéraire qu'ait la morale publique»(p.318). Et s'il ne fallait qu'une preuve de l'influence importante du roman-feuilleton sur la société du dix-neuvième siècle et de sa pénétration dans toutes ses diverses couches - faits longtemps minimisés par les historiens de la littérature - ces lettres suffisent largement à la tâche.

5 Ainsi que le souligne son éditeur - qui accomplit ici un travail énorme et méticuleux dont tous les dix-neuviémistes devront lui savoir gré - « un des principaux intérêts que présente la publication de la correspondance d'Eugène Sue est de rétablir des vérités » (p.13). Mission brillamment accomplie. La seule chose qui manque au volume est une petite phrase à la toute dernière page: "La suite à demain ». Espérons pour le plus grand plaisir des amateurs que le troisième volume de la Correspondance générale d'Eugène Sue viendra bientôt compléter la série.

\section{AUTEURS}

\section{VITTORIO FRIGERIO}

Dalhousie University 